\title{
Perceptual coding strategies in the formation and verification of descriptions*
}

\author{
HERBERT H. CLARK \\ Stanford University, Stanford, California 94305 \\ and \\ WILLIAM G. CHASE \\ Carnegie-Mellon University, Pittsburgh, Pennsylvania 15213
}

\begin{abstract}
Three experiments were carried out to study the "picture coding" process implicit both in making up descriptions of pictures and in verifying descriptions against pictures. In the first experiment, Ss were asked simply to describe pictures of one object above another; some pictures were symmetrical vertically and some were not. In the other two experiments, other Ss were timed as they judged whether sentences like Star isn't below line were true or false of such pictures. According to the results, Ss comply to three ordered "preference rules" in describing the two objects, rules that are conditional on characteristics of the picture and demands of the task. Furthermore, Ss in the verification task comply to the same three rules when they view and encode the picture before they read the sentence to be verified, but to only one of the rules when they view the picture after they read the sentence. The results also reconfirm two recently proposed models for the process of verifying sentences against pictures.
\end{abstract}

When confronted with a picture of two objects one above the other, people typically find some descriptions of that picture preferable to others, and they also find some descriptions easier than others to verify against this picture. In the case of preferences, for example, people are more likely to describe a pencil lying on a piece of paper as The pencil is on the paper than as The paper is under the pencil. And in the case of verification, for example, people can say whether or not a sentence is true or false of a picture more quickly when that sentence contains above than when it contains below (Chase \& Clark, 1971; Clark \& Chase, 1972; Seymour, 1969). But what is the relation between preferences and sentence verification? In the present paper we propose that there is a general set of coding strategies-called preference rules-by which people code simple pictures in an abstract format. The codes resulting from these rules then serve as the basis on which people construct descriptions of pictures and as the information against which they decide whether descriptions are true or false. Because the same preference rules are used in both sentence construction and sentence verification, these two processes bear a relation to each other, but the relation between them is not very direct. Our purpose is to examine one such set of preference rules and discover how they enter into the formation and verification of descriptions. To simplify our task, we have chosen visual configurations (hereafter called simply pictures) with one object located above another and descriptions

*This research was supported in part by United States Public Health Service Grants MH-07722 and MH-20021 from the National Institute of Mental Health. We thank Edward E. Smith for his helpful suggestions on the manuscript and Micheline $\mathrm{Chj}$ and Barbara Schneiderman for their assistance in the experiments. containing either the preposition above or the preposition below.

\section{FORMING DESCRIPTIONS}

How does an observer arrive at a description of an $A$ above a B (where A and B can take any number of forms)? In line with previous research (Chase \& Clark, 1971, 1972; Clark \& Chase, 1972; Clark, Carpenter, \& Just, 1973), we assume that the observer first codes such pictures in an abstract propositional format-in this case, either as $\operatorname{ABOVE}(A, B)$ or as $\operatorname{BELOW}(B, A)$. The proposition denoted as $\operatorname{ABOVE}(A, B)$ is the one that underlies the English description The $A$ is above the $B$, whereas that denoted as $\operatorname{BELOW}(B, A)$ is the one that underlies The $B$ is below the $A$. These are the only two possible codes for this restricted situation, we assume, since the previous research appears to rule out the possibility, for example, that such pictures are coded in a format that is neutral with respect to these two codes, however that might be conceived (see Clark \& Chase, 1972, p. 498). Then, as a second step, the observer (O) takes these codes, or "underlying representations," and translates them into their corresponding English surface realizations: The $A$ is above the $B$ and The $B$ is below the $A$, respectively. The process of forming descriptions, therefore, is assumed to consist of two steps: (1) constructing a perceptual code from sensory information and (2) constructing a description from that code.

Why, then, does the $O$ select $\operatorname{ABOVE}(A, B)$ as his perceptual code in some situations but BELOW $(B, A)$ as his code in others? Although the psychological literature bears very little on this question, certain linguistic facts lead us to suggest the following three ordered rules: 
Rule 1: Whenever the $O$ consciously decides to code the location of $A$ [or $B$ ] , he will code the picture as ABOVE $(\mathrm{A}, \mathrm{B})$ [or BELOW(B,A)] .

Rule 2: Whenever the $O$ perceives $B$ [or $A$ ] to be a stable, prominent point of reference, he will code the picture as $\operatorname{ABOVE}(\mathrm{A}, \mathrm{B})$ [or BELOW(B,A)].

Rule 3: Whenever neither of the above conditions holds, the 0 will code the picture as $\operatorname{ABOVE}(\mathrm{A}, \mathrm{B})$.

These rules are listed in order of priority, so that if Preference Rule 1 does not apply, Preference Rule 2 is tried; and if Preference Rule 2 does not apply, Preference Rule 3 applies by default.

The main concepts behind these rules are "located object" and "point of reference." Consider the two descriptions of a picture of an A above a B, namely, $A$ is above $B$ and $B$ is below $A$. The first describes the location of $A$ with respect to a point of reference at $B$, whereas the second does just the reverse. Thus, the question Where is $A$ ?, which queries the location of $\mathrm{A}$, is properly answered by $A$ is above $B$ but not by $B$ is below $A$. The second answer is improper because, although it refers to the same objective situation as the first, it describes the location of $B$, which is not what the question demands. We can, therefore, distinguish the roles A and B play in the locative sentence $A$ is above $B$ : A can be called the "located object," whereas B can be called the "point of reference."

Preference Rules 1, 2, and 3 differ, therefore, in how they presume the $O$ has coded the roles of $A$ and $B$ in the picture. When no other considerations apply, the $O$ is assumed to use Rule 3, coding the upper object $A$ as the located object and the lower object $B$ as the point of reference. This rule is proposed because in English upwardness is normally treated as a positive direction and downwardness as a negative direction (Clark \& Chase, 1972). The evidence, roughly, is that vertical measurements implicit in such adjectives as tall, short, high, and low all require the measurement of a located object in an upward direction from the ground, floor level, or some other lower point of reference. Thus, by choosing to code an $A$ above a $B$ as $A B O V E(A, B)$, the $O$ can preserve this normal means of coding verticality by treating the upper object $A$ as the located object and the lower object $B$ as the point of reference. Indeed, it can be argued that this asymmetry for coding verticality in English arises from a directional bias in the human perceptual apparatus, where the bias is toward perceiving objects in an upward direction from naturally occurring points of reference like the ground (Clark, 1973). In any case, Preference Rule 3 has plausible linguistic support.

The $O$ is assumed to use Preference Rule 2 when certain salient perceptual considerations override the use of Preference Rule 3. Whenever, for example, the 0 perceives $\mathrm{A}$ to be a stable, prominent object compared to $B$, he will code $A$ as the point of reference and, by default, $B$ as the located object. This will lead to the code BELOW(B,A). But just what is a "stable, prominent object"? Although we cannot offer a precise definition, we can offer several characteristic examples. Consider a paper with a long horizontal line drawn across it. It seems proper enough to speak of a dot as being above or below this line, but odd to speak of the line as being above or below the dot. The line is perceived to be a prominent, stable object, leading the $\mathrm{O}$ to code it as the point of reference. In his study of vertical location, Seymour (1969) used similar configurations as stimuli, namely, a square with a dot either above or below it. The square in these stimuli is large and prominent, serving as a natural point of reference, so it seems odd to describe the stimuli as a dot with a square either above or below it. Stability in objects can probably also arise from sequential properties of stimuli. Consider a series of letter pairs, one above the other, in which one of the two letters is always, say, an F, while the other changes from pair to pair. The $O$ would be very likely to consider the recurring letter $(\mathrm{F})$ to be a stable object, thus coding it as the point of reference and the changing letter as the object located. Informal evidence such as this attests to the plausibility of Preference Rule 2.

The $O$, however, could have reasons external to the picture itself for coding one object as the object located and the other as the point of reference. This is expressed in Preference Rule 1, which presumes that these external factors normally override the perceptual factors leading to Rule 2 or 3 . For example, the $\mathrm{O}$ might have been asked about the location of $B$. He would then have reason to code $B$ as the object located and $A$, by default, as the point of reference. This would lead to the code $\operatorname{BELOW}(\mathrm{B}, \mathrm{A})$. This could occur even though, without such a prompting question, he might perceive $B$ to be a perceptually prominent object to be coded as the point of reference. Many other external circumstances, some of which we will explore, could lead the $\mathrm{O}$ to choose to code one object instead of the other as the located object.

The purpose of Experiment I was simply to elicit descriptions for two types of vertical configurations in order to verify Preference Rules 2 and 3 listed above. These two rules lead to the prediction that Os will normally describe an A above a B, where neither A nor B is a perceptually prominent figure, with sentences containing the preposition above, not below. But this response bias should be overridden when A becomes perceptually prominent, in which case Os should use descriptions containing the preposition below.

\section{EXPERIMENT I}

\section{Method}

The Ss in Experiment I were given four vertical configurations, two symmetrical and two asymmetrical, and were asked to describe each one with a simple descriptive sentence. The two symmetrical displays consisted of a typed 
asterisk $\left({ }^{*}\right)$ one typewriter line above or below a typed lowercase $0(0)$; the two asymmetrical configurations consisted of a typed asterisk $(*)$ centered one typewriter line above or below nine typed underscores ( ). The four configurations are shown in Table 1. Each configuration was placed exactly in the middle of a mimeographed sheet, and the four sheets were given to each $S$ in one of a variety of random orders. The 92 Ss, Stanford University students run en masse in 5 min of a regular class, were instructed to "write at the bottom of each page one simple, but accurate, sentence that characterizes or describes the picture on that page."

\section{Results}

Each of the 368 descriptions was categorized as to whether it contained (1) above or one of its synonyms (e.g., over), (2) below or one of its synonyms, or (3) neither. The percentages of above and below descriptions for each configuration are shown in Table 1. First, it is clear that the two "symmetrical" configurations were described far more often with above than with below ( $\mathrm{p}<.001$, sign test, in both cases); the 72-15 split for the star above the disk was not significantly different from the 67-20 split for the disk above the star. For the two asymmetrical pictures, in contrast, whereas the star above the line was described predominantly with above ( $<<.001$, sign test), the star below the line was described predominantly with below $(p<.001$, sign test). Overall, however, the two asymmetrical configurations were still more often described with above than with below ( $\mathrm{p}<.0013$, sign test). Finally, the two symmetrical pictures were more often described overall with above than were the two asymmetrical pictures $(\mathrm{p}<.001$, sign test).

\section{Discussion}

The results of Experiment I provide clear empirical support for Preference Rules 2 and 3. First, Preference Rule 2 was supported by the results of the asymmetrical pictures in which there is a prominent stable object (the line) that could serve as the point of reference. For these pictures the use of above and below in descriptions was conditional on whether the star $\left(^{*}\right)$ was above or below the point of reference. Second, and perhaps more important, Preference Rule 3 was supported by the results for the symmetrical pictures in which there is no prominent object capable of serving as the point of reference. In this case, the pictures were generally described as $A$ is above $B$, regardless of the picture, just as stated in Preference Rule 3. Even for the asymmetrical pictures, where there was a strong perceptual point of reference, the Ss still showed a slight preference for descriptions containing above over those containing below.

To be more exact, these data can be fitted to a model implied by the ordering of Preference Rules 2 and 3 . Consider only those Ss who made use of above, below, or one of their synonyms. Let us assume that Ss will perceive the line in the asymmetrical pictures as a
Table 1

\begin{tabular}{lccr} 
Percentage of Descriptions Containing “Above” & or “Below" \\
\hline & $\star$ & 0 & \\
Symmetrical Pictures & $\mathrm{Q}$ & $\star$ & Mean \\
\hline "Above" Descriptions & 72 & 67 & 70 \\
"Below" Descripticns & 15 & 20 & 18 \\
\hline & $\star$ & - & \\
Asymmetrical Pictures & - & $\star$ & Mean \\
\hline "Above" Descriptions & 83 & 26 & 54 \\
"Below" Descriptions & 10 & 61 & 35 \\
\hline
\end{tabular}

reference point only sometimes, so the probability of using Preference Rule 2 is $p_{2}$. Let us further assume that those Ss not using Preference Rule 2 will use Preference Rule 3 and that the probability of using the code ABOVE $(A, B)$ for these $S s$ is $p_{3}$. As it turns out, this model predicts the two probabilities for the asymmetrical pictures exactly, where $\mathrm{p}_{2}=.594$ and $\mathrm{p}_{3}=.735$. If we assume that Ss invariably use Preference Rule 3 on the symmetrical pictures, we have an independent estimate of $p_{3}$, and that value is .796. Thus, the tendency to use $\mathrm{ABOVE}(\mathrm{A}, \mathrm{B})$ over $\mathrm{BELOW}(\mathrm{B}, \mathrm{A})$ is very similar for the symmetrical and asymmetrical pictures (.796 and .735 , respectively), once we eliminate those Ss using Preference Rule 2 on the asymmetrical pictures.

The tendency to use ABOVE(A,B) over BELOW(B,A) given Preference Rule 3, it should be noted, was not perfect-it was .796 and .735 on the symmetrical and asymmetrical pictures, respectively. Yet Preference Rule 3 , as written now, allows no exceptions: $p_{3}$ should be 1.00. For this experiment alone, this is not terribly important, since the rule could have been written in terms of a tendency to use $\operatorname{ABOVE}(\mathrm{A}, \mathrm{B})$ over BELOW(B,A). But for verification tasks in which Ss encode such pictures over and over again (as in Experiments II and III), it appears that Preference Rule 3 is best stated in a form with no exceptions. For this reason, we will leave the rule the way it is, remembering that there is this difference between single encodings (as in Experiment I) and repeated encodings (as in Experiments II and III). It is also noteworthy that the tendency to use $\mathrm{ABOVE}(\mathrm{A}, \mathrm{B})$ over $\mathrm{BELOW}(\mathrm{B}, \mathrm{A})$ is far greater than would be predicted simply by the frequency of occurrence of above and below in English. The ratio of $\operatorname{ABOVE}(\mathrm{A}, \mathrm{B})$ to $\mathrm{BELOW}(\mathrm{B}, \mathrm{A})$ for the symmetrical pictures in Experiment I was 3.90, while the ratio of above to below in English (Kucera \& Francis, 1967) is only 2.04 , which is about half of 3.90 .

\section{VERIFICATION OF DESCRIPTIONS}

Now that we have some evidence for how the $\mathrm{O}$ codes an $A$ above a $B$ in unstructured situations, we can turn to the process by which the $O$ verifies descriptions against his coding of such a picture. In the basic situation, the $\mathrm{O}$ is presented a sentence, like the 
schematic $A$ is above $B$, and a picture of an A above a B, and he is required to say whether the sentence is true or false of the picture. There are two possible orders in which he could carry out this task. First, he could look at the picture before reading the sentence. In this case he would code the picture, then code the sentence, then compare the two codes, and then respond with the correct answer. Since he cannot know what the sentence will be at the time he codes the picture, he will presumably code the picture according to Preference Rules 2 and 3, just as the Ss in Experiment I did. Second, he could look at the picture only after reading the sentence. In this instance, he will presumably code the sentence before coding the picture. This affords him the possibility of coding the picture according to Preference Rule 1, attempting, for example, to code the item described in the subject of the sentence as the object located and the other item as the point of reference. In short, we assume that the picture-first task should lead to evidence that the $\mathrm{O}$ has used Preference Rules 2 and 3 in coding the picture, just as if he were in the normal unstructured situation (as in Experiment I), but that the sentence-first task should show evidence of the $\mathrm{O}$ using Preference Rule 1 for coding the picture, where the picture code is somehow contingent on the sentence code that preceded it. Experiments II and III were designed to test this general hypothesis.

In several previous papers (Chase \& Clark, 1971, 1972; Clark \& Chase, 1972), :ve have already presented models for the picture-first task (Model B) and for the sentence-first task (Model A). Both models consist of four stages. At the sentence encoding stage, the $S$ represents the meaning of the sentence in a propositional format. For example, he would represent $A$ is above $B$ as A BOVE (A, B) and $A$ isn't above $B$ as FALSE(ABOVE $(A, B))$. At the picture encoding stage, he represents the picture in the same format, coding the picture ${ }_{\mathbf{B}}^{A}$ either as $\operatorname{ABOVE}(\mathrm{A}, \mathrm{B})$ or as $\mathrm{BELOW}(\mathrm{B}, \mathrm{A})$. At the comparison stage, the $S$ compares the sentence and picture representations he has constructed via a series of match-mismatch judgments and at the response stage, he converts the outcome of the comparison stage-true or false-into a motor response, e.g., a spoken "true" or "false." The main difference between Models A and B is in the ordering of the sentence representation and picture representation stages: In Model A the sentence representation stage comes first. This ordering, however, brings with it important differences in the coding of the pictures and, consequently, in the form of the comparison stage. Model B, for the picture-first task, presumes that Ss normally use Preference Rule 3 in coding the pictures. That is, Ss are assumed to invariably code ${ }_{B}^{A}$ as $\operatorname{ABOVE}(A, B)$ at the first picture representation stage. They then code the sentence in the appropriate form at the sentence representation stage. The duty of the next stage, the comparison stage, is to compute a "truth index" to indicate whether the sentence is true or false of the picture. The truth index is assumed to have two possible values-true and false-and to change from one value to the other whenever specific mismatches are found in the comparison of the sentence and picture representations. The comparison stage can be characterized by three ordered "production rules" (where Rule 0 is included merely to initialize the truth index):

Rule 0: Set the truth index equal to true.

Rule 1': If the first argument of the picture function does not match the first argument of the embedded sentence function, then transform the lat $\mathrm{ter}$ by the rule $\mathrm{ABOVE}(\mathrm{A}, \mathrm{B}) \rightarrow \mathrm{BELOW}(\mathrm{B}, \mathrm{A})$ or vice versa, whichever is appropriate.

Rule 1": If the embedded functions of sentence and picture do not match, change the truth index.

Rule 2: If the embedding functions of sentence and picture do not match, change the truth index.

To see how this process works, consider the picture ${ }_{B}^{A}$ and the true sentence $B$ isn't above $A$. The picture would be coded $\operatorname{ABOVE}(A, B)$ and the sentence FALSE(ABOVE $(B, A))$. With the truth index initialized at true by Rule 0 , Rule $1^{\prime}$ would find a mismatch between the first argument of the picture (A) and the first argument of the sentence (B) and would, therefore, transform the sentence code to FALSE(BELOW(A,B)). Rule 1 " would then apply, finding a mismatch between the $A B O V E(A, B)$ of the picture code and the BELOW $(A, B)$ of the resulting sentence code and would, therefore, force a change of the truth index from true to false. Rule 2 would then apply, finding a mismatch of the FALSE() of the sentence and the lack of this function in the picture code; this would force a change of the truth index from false back to true The final value of the truth index-true in this case-will always be the correct value and will be executed by the response stage. To predict response latencies, this model simply assumes that the separate operations in this model each take time and that these times are additive. At the sentence encoding stage, the word below takes a longer to represent than above, and negatives take $b$ longer to represent than positives. At the comparison stage, Rules $1^{\prime}, 1^{\prime \prime}$, and 2 , respectively, require $e, c$, and d increments of time whenever they are needed.

Working from Model B, we were able to show (Chase \& Clark, 1972; Clark \& Chase, 1972) that Ss presented symmetrical pictures in a picture-first verification task normally use Preference Rule 3 . There were two main pieces of evidence. First, Model B correctly predicted the observed verification latencies only if it was assumed that subjects in this picture-first task invariably coded the symmetrical picture as $\operatorname{ABOVE}(\mathrm{A}, \mathrm{B})$. Second, in a second experiment we essentially required Ss to use Preference Rule 1 and to code the top figure with respect to the bottom on some trials and to code the bottom figure with respect to the top on other trials. 
Table 2

Latency Components of Model B, Observed Mean Latencies, Predicted Mean Latencies, and Percent Error for the Seven Code Star Ss of Experiment II

\begin{tabular}{|c|c|c|c|c|c|}
\hline \multirow[b]{2}{*}{ Picture } & \multirow[b]{2}{*}{ Sentence } & \multirow[b]{2}{*}{ Latency Components } & \multicolumn{2}{|c|}{ Mean Latency } & \multirow{2}{*}{$\begin{array}{c}\text { Percent } \\
\text { Error }\end{array}$} \\
\hline & & & Observed & Predicted & \\
\hline$\star$ & $\begin{array}{l}\text { Star is above line } \\
\text { Line is below star } \\
\text { Line is above star } \\
\text { Star is below line } \\
\text { Line isn't above star } \\
\text { Star isn't below line } \\
\text { Star isn't above line } \\
\text { Line isn't below star }\end{array}$ & $\begin{array}{l}t_{0} \\
t_{0}+a+(e+q) \\
t_{0}+c+(e+q) \\
t_{0}+a+c \\
t_{0}+c+(b+d)+(e+q) \\
t_{0}+a+c+(b+d) \\
t_{0}+(b+d) \\
t_{0}+a+(b+d)+(e+q)\end{array}$ & $\begin{array}{l}1138 \\
1454 \\
1378 \\
1370 \\
1894 \\
1796 \\
1777 \\
1999\end{array}$ & $\begin{array}{l}1211 \\
1453 \\
1399 \\
1349 \\
1929 \\
1879 \\
1741 \\
1984\end{array}$ & $\begin{array}{r}2.7 \\
14.3 \\
9.8 \\
14.3 \\
28.6 \\
17.9 \\
8.0 \\
22.3\end{array}$ \\
\hline$\star$ & $\begin{array}{l}\text { Line is above star } \\
\text { Star is below line } \\
\text { Star is above line } \\
\text { Line is below star } \\
\text { Star isn't above line } \\
\text { Line isn't below star } \\
\text { Line isn't above star } \\
\text { Star isn't below line }\end{array}$ & $\begin{array}{l}t_{0}+(e+q) \\
t_{0}+a \\
t_{0}+c \\
t_{0}+a+c+(e+q) \\
t_{0}+c+(b+d) \\
t_{0}+a+c+(b+d)+(e+q) \\
t_{0}+(b+d)+(e+q) \\
t_{0}+a+(b+d)\end{array}$ & $\begin{array}{l}1367 \\
1254 \\
1328 \\
1533 \\
1793 \\
2020 \\
1884 \\
1903\end{array}$ & $\begin{array}{l}1357 \\
1307 \\
1253 \\
1495 \\
1783 \\
2026 \\
1887 \\
1837\end{array}$ & $\begin{array}{r}14.3 \\
0.0 \\
3.6 \\
8.9 \\
16.1 \\
22.3 \\
15.2 \\
10.7\end{array}$ \\
\hline
\end{tabular}

Under the former instruction, where we presumed Ss would code the picture $\operatorname{ABOVE}(\mathrm{A}, \mathrm{B})$, the results were identical to the neutral instruction of the previous experiment; under the latter instruction, where we presumed Ss would use the code BELOW(B,A), the results were radically different, though still predictable from Model B with the appropriate change in the picture code. Since the pictures in these experiments were always symmetrical, however, Ss had no opportunity to use Preference Rule 2. We now turn to Experiment II, which was devised to see whether Ss in the picture-first task would use Preference Rule 2, Preference Rule 3, or some combination of the two for asymmetrical pictures of the type used in Experiment $\mathrm{I}$.

\section{EXPERIMENT II}

\section{Method}

The Ss in this experiment were required to verify sentences like Line isn't below star against one of two asymmetrical pictures as quickly as possible while they were timed. The 16 different displays used were constructed by combining each of eight different sentences on the left with each of two pictures on the right. The eight sentences were Star is above line, Star isn't above line, Line is above star, Line isn't above star, and the same four sentences with below in place of above; the two asymmetrical pictures consisted of an asterisk either above or below a line (exactly as in Experiment I). The 16 displays, typed in elite type with the pictures 2 in. to the right of the end of the sentences, were viewed at $18 \mathrm{in}$. in a Polymetric two-field tachistoscope.

Each S was given 17 blocks of 16 trials, each block consisting of a different random order of the 16 displays; the first block was later discarded as practice. On each trial, the $S$ pressed a "ready" button, which was followed $1 \mathrm{sec}$ later by a continuous presentation of the display in the second field of the tachistoscope. The $S$ was instructed to look at the picture first, then read the sentence, and then press either a "true" or "false" button as quickly as possible. The S was timed in hundredths of a second from the moment the display lit up to the moment a button was pressed, at which time the display went off. After each trial, he was told his time if he was correct or he was told he had made an error if he was incorrect. After a short interval, the $S$ was permitted to initiate the next trial. The 282 trials were completed in less than an hour in one session. The hand-held response panel was constructed such that the $S$ rested his left and right thumbs over the "true" and "false" buttons, and he could reach the "ready" button with either thumb.

The Ss, 12 Carnegie-Mellon University summer students paid $\$ 1.50$ for their services, were urged to be as accurate as possible. while still trying to be as quick as possible. The "true" button was assigned to the right thumb for half the Ss and to the left thumb for the other half.

\section{Results}

Because the goal of this experiment was to see whether Ss would use Preference Rule 2, Preference Rule 3, or both, we attempted to divide Ss into two groups: those Ss who used Preference Rule 2 and invariably coded the star with respect to the line and those Ss who used Preference Rule 3 and invariably coded the top figure (no matter whether it was a star or line) with respect to the bottom figure. The first group will be called code star Ss and the latter code top Ss. The latencies predicted by Model B for the code star Ss consist of the addition of increments $t_{0}, a, b, c, d, e$, and $q$ in the pattern shown in Table 2 for each of the 16 displays. As noted above, a is added whenever the sentence contains below; b is added whenever the sentence contains a negative; and $e, c$, and $d$ are added whenever Rules $1^{\prime}, 1^{\prime \prime}$, and 2 , respectively, are required. These are all added to a base time $t_{0}$, which is a measure of the processes (encoding, responding, etc.) common to all the conditions. In addition, we have included parameter $q$ for the possibility that it takes an increment $q$ longer to encode a sentence with line as the subject than with star as the subject; a similar increment was required in Experiments 1,2, and 3 of Clark and Chase (1972). 
Table 3

Latency Components of Model B, Observed Mean Latencies, Predicted Mean Latencies, and Percent Error for the Five Code Top Ss of Experiment II

\begin{tabular}{|c|c|c|c|c|c|}
\hline \multirow[b]{2}{*}{ Picture } & \multirow[b]{2}{*}{ Sentence } & \multirow[b]{2}{*}{ Latency Components } & \multicolumn{2}{|c|}{ Mean Latency } & \multirow{2}{*}{$\begin{array}{r}\text { Percent } \\
\text { Error }\end{array}$} \\
\hline & & & Observed & Predicted & \\
\hline$\star$ & $\begin{array}{l}\text { Star is above line } \\
\text { Line is below star } \\
\text { Line is above star } \\
\text { Star is below line } \\
\text { Line isn't above star } \\
\text { Star isn't below line } \\
\text { Star isn't above line } \\
\text { Line isn't below star }\end{array}$ & $\begin{array}{l}t_{0} \\
t_{0}+a+e+q \\
t_{0}+c+e+q \\
t_{0}+a+c \\
t_{0}+c+(b+d)+e+q \\
t_{0}+a+c+(b+d) \\
t_{0}+(b+d) \\
t_{0}+a+(b+d)+e+q\end{array}$ & $\begin{array}{l}1451 \\
1823 \\
1771 \\
1733 \\
2612 \\
2453 \\
2164 \\
2568\end{array}$ & $\begin{array}{l}1445 \\
1825 \\
1818 \\
1711 \\
2566 \\
2460 \\
2193 \\
2573\end{array}$ & $\begin{array}{r}3.8 \\
10.0 \\
1.2 \\
5.0 \\
17.5 \\
13.8 \\
7.5 \\
13.8\end{array}$ \\
\hline$\star$ & $\begin{array}{l}\text { Line is above star } \\
\text { Star is below line } \\
\text { Star is above line } \\
\text { Line is below star } \\
\text { Star isn't above line } \\
\text { Line isn't below star } \\
\text { Line isn't above star } \\
\text { Star isn't below line }\end{array}$ & $\begin{array}{l}t_{0}+q \\
t_{0}+a+e \\
t_{0}+c+e \\
t_{0}+a+c+q \\
t_{0}+c+(b+d)+e \\
t_{0}+a+c+(b+d)+q \\
t_{0}+(b+d)+q \\
t_{0}+a+(b+d)+e\end{array}$ & $\begin{array}{l}1547 \\
1722 \\
1760 \\
1790 \\
2448 \\
2541 \\
2290 \\
2508\end{array}$ & $\begin{array}{l}1533 \\
1737 \\
1730 \\
1799 \\
2478 \\
2547 \\
2281 \\
2486\end{array}$ & $\begin{array}{r}8.8 \\
8.8 \\
3.8 \\
5.0 \\
10.0 \\
21.2 \\
5.0 \\
13.8\end{array}$ \\
\hline
\end{tabular}

It should be noted that, since $b$ and $d$ occur together whenever they occur, they must be treated as a single parameter $(b+d)$. This is also true of $e$ and $q$, which must be treated as a single parameter $(e+q)$. The latency components predicted by Model $B$ for the code top Ss are shown in Table 3. The only difference between the code top and the code star Ss is found in the parameter e. For the code top Ss, e is orthogonal to $q$ and must, therefore, be treated as a separate parameter.

To determine which Ss were code star Ss and which were code top Ss, we performed a preliminary analysis of variance on each S separately. Each analysis consisted of a Picture by Polarity by Truth by Preposition ( 2 by 2 by 2 by 2) factorial design with repeated neasures; each analysis made use of only the correct responses. As Tables 2 and 3 indicate, if a $S$ is a code star $S$, the Picture by Polarity by Truth by Preposition interaction effect should be larger than the Picture by Polarity by Preposition interaction effect; but if a $S$ is a code top $S$, the reverse should be true. By this method, seven Ss were classified as code star Ss and five as code top Ss. Indeed, the four-way interaction effect was significantly greater than zero (and in the right direction) for every $S$ in the code star group but for none of the Ss in the code top group; likewise, the appropriate three-way interaction effect was significantly greater than zero (and in the right direction) for four of the five code top Ss but for only one code star $\mathbf{S}$. Although this one $\mathrm{S}$ may have used both strategies during the course of the experiment, we will treat him as a code star $S$.

Tables 2 and 3 show the observed mean latencies and error percentages for each of the 16 displays for the code star and code top Ss separately. These tables also give the mean latencies predicted by Model B. The observed mean latencies were calculated by finding the mean latency of the 16 or fewer correct responses for each display of each subject and then taking the average of these means across Ss. The predicted mean latencies were calculated by determining the least-squares estimates of each parameter from the observed mean latencies and then inserting these values in the appropriate formulae in Tables 2 and 3. Table 4 gives the least-squares estimates (in milliseconds) for the five parameters of the code star Ss, for the six parameters of the code top Ss, and for the five parameters of all the Ss in a comparable picture-first task (Experiment 2) in Clark and Chase (1972), where the pictures were symmetrical. Table 4 also gives the root mean squared deviations (RMSD) for each model (see Sternberg, 1969).

There are several steps in assessing whether or not Model B provides a good fit to the data in Tables 2 and 3. First, one should demonstrate that each parameter estimate is significantly greater than zero. This can be done by referring to a Picture by Polarity by Truth by Preposition ( 2 by 2 by 2 by 2 ) analysis of variance for the display means of the code star Ss and a similar one for the display means of the code top Ss. In these analyses, for example, if the preposition mean effect is

Table 4

Parameter Estimates for the Top and Star Groups of Experiment II and from Experiment II of Clark and Chase (1972)

\begin{tabular}{cccc}
$\begin{array}{c}\text { Para- } \\
\text { meters }\end{array}$ & $\begin{array}{c}\text { Clark } \\
\text { \& Chase } \\
\text { Experiment II }\end{array}$ & $\begin{array}{c}\text { Code } \\
\text { Star Ss }\end{array}$ & $\begin{array}{c}\text { Code } \\
\text { Top Ss }\end{array}$ \\
\hline $\mathrm{t}_{0}$ & 1793 & 1211 & 1445 \\
$\mathrm{a}$ & 128 & 96 & 137 \\
$\mathrm{c}$ & 91 & 42 & 129 \\
$\mathrm{e}$ & 212 & $(146)^{*}$ & 155 \\
$\mathrm{q}$ & -- & 531 & 88 \\
$(\mathrm{~b}+\mathrm{d})$ & 504 & 43.5 & 748 \\
RMSD & 39.2 & & 23.3 \\
\hline
\end{tabular}

$*(e+q)$ 
significant, this is equivalent to showing that a is significantly greater than zero. Similarly, the parameter $(b+d)$ corresponds to the Polarity by Truth interaction and, in general, each parameter corresponds to a 1-df main effect or interaction effect in these analyses of variance. Thus, for the code star group the parameters a, $(b+d)$, c, and $(e+q)$ resulted in $F(1,6) s$ of $11.3,22.1$, 9.58, and 103.3, respectively, all significant with at least $p<.05$. For the code top group the parameters a, $(b+d), c, e$, and $q$ resulted in $F(1,4) s$ of $4.34,10.2$, $30.5,9.78$, and 16.8 , respectively. For this group, only parameter a failed to reach significance $(p<.05)$, but since the magnitude of a (137 msec) is in the appropriate range (see Clark \& Chase, 1972), this failure was probably due solely to the low power of the significance test here. Second, one must demonstrate that there are no other significant main effects or interactions in these two analyses of variance, for if there were, that would indicate nonadditivity in the parameters, contrary to Model B. Indeed, none of the remaining effects in either analysis approached significance. Finally, one can examine how closely the predicted mean latencies approximate the observed mean latencies. For the code star group, the model in Table 2, with four parameters (excluding $t_{0}$ ), accounts for $97.6 \%$ of the variance among the 16 means; the error in the model, as indicated by the RMSD of $43.5 \mathrm{msec}$ with $11 \mathrm{df}$, was not significant. For the code top group, the model in Table 3, with five parameters, accounted for $99.6 \%$ of the variance among the 16 means; the RMSD of $23.3 \mathrm{msec}$ with $10 \mathrm{df}$ was not significant. In sum, Model $\mathrm{B}$ gives an excellent fit to the data.

The error rate was $13.1 \%$ for the code star Ss and 9.3\% for the code top Ss. The errors showed the same pattern as the latencies. The correlation between the observed latencies and the percent error for the 16 displays was .74 for the code star Ss and .75 for the code top Ss. Furthermore, there was an increase in errors correlated with each of the four parameters in the model for the code star Ss and with each of the five parameters in the model for the code top Ss. This suggests that the errors are produced by the same process that leads to increased latencies, and it helps to rule out any substantial latency-error trade-off that might confound the latencies.

\section{Discussion}

The first important result of Experiment II is that Ss appear to follow Preference Rules 2 and 3 in approximately the same proportion no matter whether they are verifying pictures in a picture-first verification task or describing them in a free situation (like Experiment I). Consider the asymmetrical pictures. In the verification task of Experiment II, 7/12 or .56 of the Ss used Preference Rule 2, coding the star with respect to the line; the remainder used Preference Rule 3, coding the top with respect to the bottom. This .56 proportion corresponds almost exactly to the .59 proportion of Ss in Experiment 1 who used Preference Rule 2 in their descriptions of these same pictures; the remaining $S s$ in that experiment again used Preference Rule 3. The results with asymmetrical pictures contrast sharply with those with symmetrical pictures. In the picture-first verification task of Clark and Chase's Experiment 2, Ss invariably coded the symmetrical pictures by Preference Rule 3; this corresponds directly to the $\mathrm{Ss}$ in the present Experiment I who invariably described the same pictures by use of Preference Rule 3. It is especially significant that no Ss were found in the present experiment who consistently used the opposites of Preference Rules 2 and 3 ; that is, no $S$ was found who invariably encoded the large stable object (the line) with respect to the other object (the star) or who invariably encoded the bottom figure with respect to the top one. All this evidence, then, demonstrates that Preference Rules 2 and 3 are quite accurate in accounting for both (1) the descriptions Ss will construct for symmetrical and asymmetrical pictures when given freedom of choice and (2) the way Ss will encode these pictures in verification tasks where the picture is viewed first.

A second important result is that the latencies for asymmetrical pictures are accurately accounted for by Model B. The code star and code top strategies of Experiment II have relatively simple consequences on parameter e of Model B, and, once these consequences are taken into consideration, this single model accounts nicely for all the Ss. The magnitude of the parameters in Experiment II are also in good agreement with the previous findings of Clark and Chase (see Table 4). In all, therefore, Model B has been shown to work for picture-first verification tasks with symmetrical pictures (Experiment 2, Clark \& Chase, 1972), asymmetrical pictures (the present Experiment II), and symmetrical pictures with Ss instructed to focus either on the top or the bottom of the picture (Experiment 3, Clark \& Chase, 1972).

Finally, it was found that Ss do not take reliably longer to encode the star as being below the line than as being above the line. This difference (the picture main effect in the two analyses of variance) was not significant, and it averaged only $35 \mathrm{msec}$. This finding agrees quite well with the comparable outcome of Experiment 2 in Clark and Chase (1972), where it was found that Ss took a nonsignificant $15 \mathrm{msec}$ longer to represent symmetrical pictures when they focused their attention on the bottom than when they focused their attention on the top.

It is hardly surprising, perhaps, that picture asymmetries affect picture-first verification tasks in the same way they do free descriptions. After all, the S codes the picture in both tasks before he does anything else. The critical issue, therefore, is how picture asymmetries affect the picture encoding stage in a sentence-first verification task. We now turn to Experiment III, which was devised tu investigate just this issue. 
Table 5

Sentence-Picture Combinations of Experiment III and Latency Components Predicted by Model $\mathbf{A}+(\mathrm{e}+\mathrm{q})+\mathbf{p}$

\begin{tabular}{lll}
\hline \multicolumn{1}{c}{ Sentence } & Picture & \multicolumn{1}{c}{ Latency Components } \\
\hline Star is above line & $t_{0}$ \\
Line is below star & & $t_{0}+a+(e+q)+p$ \\
Line is above star & $t_{0}+c+(e+q)$ \\
Star is below line & $\star$ & $t_{0}+a+c+p$ \\
Line isn't above star & & $t_{0}+c+(b+d)+(e+q)$ \\
Star isn't below line & & $t_{0}+a+c+(b+d)+p$ \\
Star isn't above line & & $t_{0}+(b+d)$ \\
Line isn't below star & & $t_{0}+a+(b+d)+(e+q)+p$ \\
Line is above star & & $t_{0}+(e+q)+p$ \\
Star is below line & & $t_{0}+a$ \\
Star is above line & $t_{0}+c+p$ \\
Line is below star & $\quad$ & $t_{0}+a+c+(e+q)$ \\
Star isn't above line & $t_{0}+c+(b+d)+p$ \\
Line isn't below star & & $t_{0}+a+c+(b+d)+(e+q)$ \\
Line isn't above star & $t_{0}+(b+d)+(e+q)+p$ \\
Star isn't below line & $t_{0}+a+(b+d)$ \\
\hline
\end{tabular}

\section{EXPERIMENT III}

In our earlier work (Chase \& Clark, 1972; Clark \& Chase, 1972), we had proposed Model A to account for the process by which the $\mathrm{S}$ verifies sentences such as Plus isn't above star in a sentence-first verification task with symmetrical pictures. Model A is very much like Model B but with several important differences. At the sentence encoding stage, the $\mathrm{S}$ first encodes the sentence in exactly the same form as in Model B. At the picture encoding stage, however, the $S$ codes the picture contingent on the preposition encountered in the sentence he has just read. If the sentence contained above, he codes the picture as $\mathrm{ABOVE}(\mathrm{A}, \mathrm{B})$; but if the sentence contained below, he codes the picture as BELOW(B,A). This contingent coding scheme is quite unlike its counterpart in Model $B$. The comparison stage of Model A, therefore, requires only two rules (in addition to the initializing rule):

Rule 0: Set the truth index equal to true.

Rule 1: If the first argument of the picture function does not match the first argument of the embedded sentence function, change the truth index.

Rule 2: If the embedding functions of sentence and picture do not match, change the truth index.

Thus, because the sentence and picture always contain the same preposition, Rules $1^{\prime}$ and 1 " of Model B can be reduced to the rather simple Rule 1 for Model A. Model A, therefore, requires one less parameter than Model B. Below still takes a longer to encode than above, and negatives still take $b$ longer to encode than positives, but Rules 1 and 2 of the comparison stage require $c$ and $d$ increments of time, respectively, whenever they are required. So, while Model B had parameters $a,(b+d), c$, and e, Model $A$ has only the first three of these, $a,(b+d)$, and $c$. This model was shown to be extremely accurate in predicting latencies in a sentence-first task with symmetrical pictures (Experiment 1, Clark \& Chase, 1972).

As Model $\mathrm{A}$ is now formulated, the $\mathrm{S}$ codes the picture by a contingent coding scheme, and this is equivalent to Preference Rule 1 . That is, the $S$ is not free to code the picture according to Preference Rule 2 or 3 because, once he has read the sentence, the preposition it contains determines how he will code the picture. But when the picture is asymmetrical, Model A might not be so appropriate. There are essentially three ways in which asymmetrical pictures could affect the process.

The first possibility is that the asymmetry will have no effect on the picture coding stage at all: The $S$ would invariably use Preference Rule 1 no matter whether the pictures were symmetrical or asymmetrical. That is, the $\mathrm{S}$ would invariably code the picture as $\operatorname{ABOVE}(\mathrm{A}, \mathrm{B})$ when the sentence had contained above and as BELOW(B,A) when the sentence had contained below, just as Model A says he should do. In this case, the verification latencies should be well accounted for by Model A and its three parameters-a, $(b+d)$, and c. As we noted before, however, there is another parameter that should enter here. Parameter $q$ is the possible extra time the $S$ requires to encode line as the subject of the sentence over the time he requires to encode star. This first possible model will, therefore, be called Model $\mathrm{A}+\mathrm{q}$. The latency components predicted by this model for the 16 possible displays to be used in Experiment III can be seen in Table 5 by setting parameters $e$ and $p$ equal to zero.

The second possibility is that Preference Rule 2 will dominate Preference Rule 1 at the picture encoding stage, so that the sentence no longer determines the way in which the picture is encoded--as Model A would have it. With Preference Rule 2 taking precedence, the $\mathrm{S}$ would code the star with respect to the line no matter what sentence he had just read. If this were the case, Model B with its four parameters a, c, $(b+d)$, and $(\mathrm{e}+\mathrm{q})$ would be appropriate, since it is designed to handle just such situations in which the picture encoding stage is independent of the sentence encoding stage. The components of latency for this model can also be seen in Table 5 by setting parameter $p$ equal to zero. As an examination of Table 5 shows, however, the parameter $(e+q)$ of Model B has the same consequences on the latencies as parameter $q$ of Model $A$, so the second possibility (Model B) is indistinguishable at this point from the first possibility (Model $A+q$ ). For now we will treat this compound parameter $(e+q)$ in a model called Model $\mathrm{A}+(\mathrm{e}+\mathrm{q})$.

The third possibility is that Preference Rule 1 takes precedence at the sentence encoding stage, just as Model A suggests, but the description preferences (as reflected in Preference Rule 2) would determine the relative encoding difficulty of the two asymmetrical pictures. For example, sentences containing above and below would always induce the $S$ to encode the picture 
Table 6

Sentences and Pictures Combined, Observed Mean Latencies, Predicted Mean Latencies, and Percent Error for Experiment III

\begin{tabular}{|c|c|c|c|c|c|}
\hline \multirow[b]{2}{*}{ Sentence } & \multirow[b]{2}{*}{ Picture } & \multirow{2}{*}{$\begin{array}{c}\text { Observed } \\
\text { Mean } \\
\text { Latency }\end{array}$} & \multirow[b]{2}{*}{$\begin{array}{c}\text { Percent } \\
\text { Error }\end{array}$} & \multicolumn{2}{|c|}{ Predicted Latency } \\
\hline & & & & $\begin{array}{r}\text { Model A } \\
+(e+q) \\
\end{array}$ & $\begin{array}{l}\text { Model A+ } \\
(e+q)+p\end{array}$ \\
\hline Star is above line & & 1175 & 3.3 & 1233 & 1211 \\
\hline Line is below star & & 1486 & 10.0 & 1448 & 1470 \\
\hline Line is above star & & 1471 & 9.2 & 1468 & 1447 \\
\hline Star is below line & $\star$ & 1526 & 6.7 & 1524 & 1545 \\
\hline Line isn't above star & 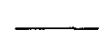 & 2079 & 28.3 & 2139 & 2117 \\
\hline Star isn't below line & & 2214 & 34.2 & 2194 & 2216 \\
\hline Star isn't above line & & 1924 & 13.3 & 1904 & 1882 \\
\hline Line isn't below star & & 2136 & 17.5 & 2119 & 2140 \\
\hline Line is above star & & 1356 & 10.8 & 1313 & 1334 \\
\hline Star is below line & & 1377 & 9.2 & 1368 & 1347 \\
\hline Star is above line & & 1418 & 0.8 & 1388 & 1409 \\
\hline Line is below star & - & 1536 & 9.2 & 1604 & 1582 \\
\hline Star isn't above line & $\star$ & 2092 & 26.7 & 2059 & 2080 \\
\hline Line isn't below star & & 2313 & 25.8 & 2274 & 2253 \\
\hline Line isn't above star & & 1971 & 11.7 & 1984 & 2005 \\
\hline Star isn't below line & & 1982 & 17.5 & 2039 & 2018 \\
\hline
\end{tabular}

of a star above a line as ABOVE(STAR,LINE) and BELOW(LINE,STAR), respectively, just as Model A requires, but, since the former code is preferred to the latter (see Experiment I), the former will be easier and faster to construct at the picture encoding stage than the latter. The extra time consumed in the construction of this nonpreferred picture code will be given by parameter $\mathrm{p}$, and now a modified Model $\mathrm{A}+\mathrm{q}$ with five parameters is required. For convenience, this model will be called Model $\mathrm{A}+(\mathrm{e}+\mathrm{q})+\mathrm{p}$, and its components of latency are shown in Table 5 , where none of the parameters should be set equal to zero.

Experiment III, a sentence-first verification task with asymmetrical pictures, should, therefore, conform to one of three models: Model $A+q$, Model $A+(e+q)$, or Model $A+(e+q)+p$. Since the first two models are indistinguishable, this experiment will test only two models, Model $A+(e+q)$ and Model $A+(e+q)+p$. If the experiment turns out to support Model $A+(e+q)$, then we can examine its results relative to Experiment II in order to differentiate the first two models.

\section{Method}

Experiment III was identical to Experiment II, except that Ss were instructed to read the sentence before looking at the picture, and they were given 11 blocks of trials (the first one considered practice) rather than 17 . The Ss were 12 new Carnegie-Mellon University summer students from the same pool as those in Experiment II.

\section{Results}

Table 6 shows the observed mean latencies and error percentages for each of the 16 displays of Experiment III; it also gives the predicted mean latencies for Model $\mathrm{A}+(\mathrm{e}+\mathrm{q})$ and Model $\mathrm{A}+(\mathrm{e}+\mathrm{q})+\mathrm{p}$. As in Experiment II, the observed means were calculated by finding the mean latency for the 10 or fewer correct responses for each display for each $\mathrm{S}$ and then averaging these means over Ss. Similarly, the predicted mean latencies were calculated by determining the least-squares estimates of each parameter from the observed means and then inserting these values in the appropriate formulae. Table 7 gives the least-squares values of the parameters for Model $A$ as applied to Clark and Chase's Experiment 1, for Model $A+(e+q)$ and for Model $A+(e+q)+p$, and gives the RMSD for each model. As in Experiment II, the 16 display means from all 12 Ss were analyzed in a single Picture by Polarity by Truth by Preposition analysis of variance, and all the statistics presented below are based on this analysis, following the reasoning given in Experiment II.

There are two important findings. First, the parameter $(e+q)$ was a significant $80 \mathrm{msec}$, since the four-way interaction was significant $[F(1,11)=8.30, p<.025]$. That is, Model $\mathrm{A}+(\mathrm{e}+\mathrm{q})$ gave a better fit than Model $\mathrm{A}$ with $(e+q)=0$. Model $A$ alone, with parameters $a, c$, and $(b+d)$, accounted for $97.6 \%$ of the variance among the 16 observed mean latencies, whereas Model $\mathrm{A}+(\mathrm{e}+\mathrm{q})$, with its additional parameter $(\mathrm{e}+\mathrm{q})$, accounted for $98.9 \%$ of the variance; the RMSD was significantly reduced from 55 to $37.8 \mathrm{msec}$ by the addition of the parameter $(e+q)$. Second, the estimate

Table 7

Parameter Estimates for the Models of Experiment III and from Experiment 1 of Clark and Chase (1972)

\begin{tabular}{cccc} 
Parameters & $\begin{array}{c}\text { Clark } \\
\text { \& Chase } \\
\text { Model A }\end{array}$ & $\begin{array}{c}\text { Model A } \\
+(\mathrm{e}+\mathrm{q})\end{array}$ & $\begin{array}{c}\text { Model A } \\
+(\mathrm{e}+\mathrm{q})+\mathrm{p}\end{array}$ \\
\hline $\mathrm{t}_{\mathrm{o}}$ & 1763 & 1233 & 1211 \\
$\mathrm{a}$ & 93 & 136 & 136 \\
$\mathrm{c}$ & 187 & 155 & 155 \\
$(\mathrm{~b}+\mathrm{d})$ & 685 & 671 & 671 \\
$(\mathrm{e}+\mathrm{q})$ & -- & 80 & 80 \\
$\mathrm{p}$ & - & -- & 43 \\
RMSD & 16.0 & 37.8 & 31.2 \\
\hline
\end{tabular}


of encoding difficulty $\mathrm{p}$ was a nonsignificant $43 \mathrm{msec}$ $[F(1,11)=2.73]$, an amount only slightly larger than the RMSD. Adding parameter $p$ to Model $\mathrm{A}+(\mathrm{e}+\mathrm{q})$ did not improve the fit. As one can also see from Table 7 , the parameters were in good agreement with Experiment 1 of Clark and Chase. It should be noted that the RMSD of $37.8 \mathrm{msec}$ here is based on $11 \mathrm{df}$, whereas the RMSD of $16 \mathrm{msec}$ from Clark and Chase is based on $4 \mathrm{df}$, so the estimates of error in these two instances are more comparable than they first appear.

The rest of the results also bear out the implications of Model $\mathrm{A}+(\mathrm{e}+\mathrm{q})$. From the analysis of variance, it was found that the parameters $a, c,(b+d)$, and $(e+q)$ were all significantly greater than zero at at least $p<.05$, with $F(1,11)$ s of $16.5,5.88,58.3$, and 8.30 , respectively. Furthermore, none of these parameters interacted significantly with each other or with any other factors in the experiment, just as predicted by the additivity assumptions of the model.

The overall error rate in Experiment III was 14.6\%. As in Experiment II, the errors and mean latencies on the 16 displays were highly correlated $(r=.86)$, and there was an increase in errors associated with each of the four parameters in Model $\mathrm{A}+(\mathrm{e}+\mathrm{q})$. So, although Model $A+(e+q)$ was not designed to account for errors, it can do so roughly with the assumption that the longer the process, the more likely the $S$ is to make an error.

\section{Discussion}

Taken alone, Experiment III is consistent both with Model $\mathrm{A}+\mathrm{q}$ and with Model B, that is, with Model $A+(e+q)$. When evidence from Experiment II is also brought to bear, however, it appears that the correct model for this task is Model A + q. Note that for the crsde top $S s$ in Experiment II parameter q-the difference between line and star in the sentence encoding stage-was not confounded with $e$. The observed value of $q(88 \mathrm{msec})$ was very close to the value of the alleged $(\mathrm{e}+\mathrm{q})(80 \mathrm{msec})$ of Experiment III, and it was also in the same range as similar encoding effects in Clark and Chase. Furthermore, the e effect of the code top Ss $(155 \mathrm{msec})$ and the $(\mathrm{e}+\mathrm{q})$ effect of the code star Ss $(146 \mathrm{msec})$ in Experiment II were both considerably larger than the alleged $(e+q)$ effect in Experiment III $(80 \mathrm{msec})$, as were the $\mathrm{e}$ effects in Experiment 2 $(212 \mathrm{msec})$ and Experiment $3(196 \mathrm{msec})$ of Clark and Chase (1972). The implication is that the alleged $(e+q)$ of Experiment III was not truly $(e+q)$ at all, but rather $q$, with $e=0$. Hence, the Ss in Experiment III must have been encoding the picture contingent on the sentence they had just read, just as Model $\mathrm{A}+\mathrm{q}$ asserts.

\section{CONCLUSIONS}

In the Introduction, we suggested that people code vertically arranged objects according to three ordered rules, Preference Rules 1,2, and 3. We based these rules in the main on such linguistic considerations as the asymmetry in English of the verticality dimension, the asymmetry in English of descriptions of location, and the properties of proper answers to questions. The proposal was that these preference rules would then play a part in two rather different processes: the formation of descriptions of vertically arranged objects and the verification of such descriptions against pictures. In Experiment I we examined the descriptions people gave to symmetrical and asymmetrical pictures, and these conformed quite well with Preference Rules 2 and 3 and their ordering. In Experiments II and III, we measured the time people took to verify such descriptions, and these latencies in combination with previous results showed quite precisely when and how these preference rules are used in the process of verification.

Ss appear to be able to use all three preference rules in tasks where they view the picture before reading the sentence. Preference Rule 3, which states that with no other constraints people will choose to code the top object with respect to the bottom one, i.e., as $\operatorname{ABOVE}(A, B)$, was demonstrated most strikingly in Experiment 2 by Clark and Chase, a picture-first verification task. Presented symmetrical pictures and unconstrained by specific viewing instructions, those $\mathrm{Ss}$ coded all pictures as $\mathrm{ABOVE}(\mathrm{A}, \mathrm{B})$, not as $\mathrm{BELOW}(\mathrm{B}, \mathrm{A})$. Even in the present Experiment II, where the pictures were asymmetrical, some Ss-the five code top Ss-consistently coded all pictures as $\operatorname{ABOVE}(\mathrm{A}, \mathrm{B})$. Preference Rule 2, which states that Ss will code one object with respect to a stable prominent object (the point of reference), was consistently used by the seven code star Ss of Experiment II, who always coded the star with respect to the stabler, more prominent line. The use of Preference Rule 1, which states that Ss can decide a priori which object they will code with respect to the other, was evident in Clark and Chase's Experiment 3. In that experiment, the Ss instructed to attend to the top object were able to code the picture as $\operatorname{ABOVE}(A, B)$, and those instructed to attend to the bottom object as BELOW(B,A). In short, Ss in these picture-first tasks can make use of Preference Rules 1, 2, and 3 as the experimental conditions dictate.

Ss in the sentence-first tasks, however, appear unwilling or unable to use any rule except Preference Rule 1. In Clark and Chase's Experiment 1, the Ss coded the pictures as $\operatorname{ABOVE}(A, B)$ or $\operatorname{BELOW}(B, A)$, depending on whether the sentence they had just read contained above or below, respectively. Even in the present Experiment III, where the pictures were asymmetrical and would normally be coded quite differently (see Experiment I), the Ss still coded the pictures according to this scheme. In the sentence-first verification tasks, then, Ss are apparently forced by some rather well established strategy to replace their natural coding preferences (Preference Rules 2 and 3 ) by a contingent coding scheme (Preference Rule 1).

As ought to be expected, therefore, the construction 
and verification of descriptions of pictures have certain processes in common. The basic one is that the $\mathrm{S}$ has to choose how he will abstractly code the picture. His choice can be affected by a priori decisions as to what to code by inherent symmetries or asymmetries in the picture and even by a default decision rule in case no other decision rule applies. That is, there appears to be a hierarchy of strategies people rely on for the coding of visual information. This conception of perceptual coding leads to two very general questions: What are the strategies? And how are they organized? Our attempt at specifying Preference Rules 1,2, and 3 and their application in descriptions and sentence verification is only a small start in answer to such questions.

\section{REFERENCES}

Chase, W. G., \& Clark, H. H. Semantics in the perception of verticality. British Journal of Psychology, 1971, 62, 311-326. Chase, W. G., \& Clark, H. H. Mental operations in the comparison of sentences and pictures. In L. Gregg (Ed.), Cognition in learning and memory. New York: Wiley, 1972.

Clark, H. H. Space, time, semantics, and the child. In T. E. Moore (Ed.), Cognitive development and the acquisition of language. New York: Academic Press, 1973.

Clark, H. H., Carpenter, P. A., and Just, M. A. On the meeting of semantics and perception. In W. G. Chase (Ed.), Visual information processing. New York: Academic Press, 1973.

Clark, H. H., \& Chase, W. G. On the process of comparing sentences against pictures. Cognitive Psychology, 1972, 3, 472-517.

Kucera, H., \& Francis, W. N. Computationa! analysis of present-day American English. Providence, R.I: Brown University Press, 1967.

Seymour, P. H. K. Response latencies in judgments of spatial location. British Journal of Psychology, 1969, 60, 31-39.

Sternberg, S. The discovery of processing stages: Extensions of Donders' method. In W. G. Koster (Ed.), Attention and performance II. Acta Psychologica, 1969, 30, 276-315.

(Received for publication May 16, 1973; accepted June 11, 1973.) 